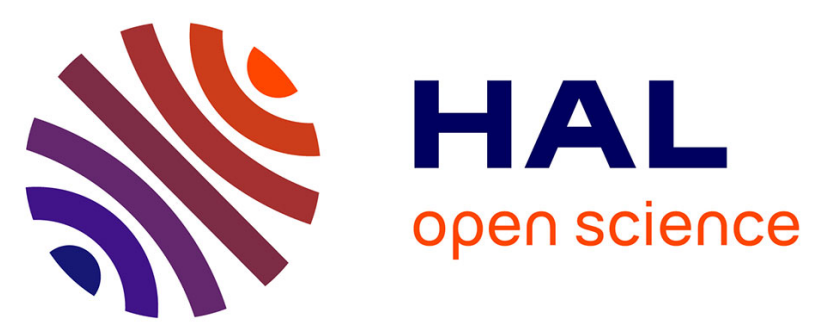

\title{
Analysis of membrane interactions of antibiotic peptides using ITC and biosensor measurements
}

Saad Al-Kaddah, Katrin Reder-Christ, Gabriela Klocek, Imke Wiedemann, Manuela Brunschweiger, Gerd Bendas

\section{- To cite this version:}

Saad Al-Kaddah, Katrin Reder-Christ, Gabriela Klocek, Imke Wiedemann, Manuela Brunschweiger, et al. Analysis of membrane interactions of antibiotic peptides using ITC and biosensor measurements. Biophysical Chemistry, 2010, 152 (1-3), pp.145. 10.1016/j.bpc.2010.09.002 . hal-00694292

\section{HAL Id: hal-00694292 \\ https://hal.science/hal-00694292}

Submitted on 4 May 2012

HAL is a multi-disciplinary open access archive for the deposit and dissemination of scientific research documents, whether they are published or not. The documents may come from teaching and research institutions in France or abroad, or from public or private research centers.
L'archive ouverte pluridisciplinaire HAL, est destinée au dépôt et à la diffusion de documents scientifiques de niveau recherche, publiés ou non, émanant des établissements d'enseignement et de recherche français ou étrangers, des laboratoires publics ou privés. 


\section{Accepted Manuscript}

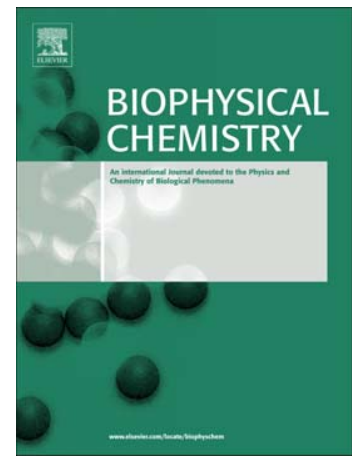

Analysis of membrane interactions of antibiotic peptides using ITC and biosensor measurements

Saad Al-Kaddah, Katrin Reder-Christ, Gabriela Klocek, Imke Wiedemann, Manuela Brunschweiger, Gerd Bendas

PII: $\quad$ S0301-4622(10)00226-7

DOI: $\quad$ doi: $10.1016 /$ j.bpc.2010.09.002

Reference: $\quad$ BIOCHE 5428

To appear in: Biophysical Chemistry

Received date: 14 July 2010

Revised date: 15 September 2010

Accepted date: 15 September 2010

Please cite this article as: Saad Al-Kaddah, Katrin Reder-Christ, Gabriela Klocek, Imke Wiedemann, Manuela Brunschweiger, Gerd Bendas, Analysis of membrane interactions of antibiotic peptides using ITC and biosensor measurements, Biophysical Chemistry (2010), doi: $10.1016 /$ j.bpc.2010.09.002

This is a PDF file of an unedited manuscript that has been accepted for publication. As a service to our customers we are providing this early version of the manuscript. The manuscript will undergo copyediting, typesetting, and review of the resulting proof before it is published in its final form. Please note that during the production process errors may be discovered which could affect the content, and all legal disclaimers that apply to the journal pertain. 


\section{Analysis of membrane interactions of antibiotic peptides using ITC and}

\section{biosensor measurements}

Saad Al-Kaddah ${ }^{\text {a }}$, Katrin Reder-Christ ${ }^{\text {a }}$, Gabriela Klocek ${ }^{\mathrm{b}}$, Imke Wiedemann ${ }^{\mathrm{c}}$,

Manuela Brunschweiger ${ }^{\mathrm{a}}$, Gerd Bendas ${ }^{\mathrm{a}} *$

${ }^{a}$ Department of Pharmacy, Rheinische Friedrich Wilhelms University Bonn, An der Immenburg 4, 53121 Bonn, Germany

${ }^{\mathrm{b}}$ Department of Biophysical Chemistry, Biozentrum, University of Basel, Klingelbergstrasse 50/70, 4056 Basel, Switzerland

${ }^{\mathrm{c}}$ Institute of Medical Microbiology, Immunology and Parasitology, Pharmaceutical Microbiology Unit, Rheinische Friedrich-Wilhelms-University Bonn, Meckenheimer Allee 168, 53115 Bonn, Germany

*To whom correspondence should be addressed: University of Bonn

Department of Pharmacy

An der Immenburg 4

53121 Bonn

Germany

Phone: +49228735250

Fax: +49228 734692

e-mail: gbendas@uni-bonn.de: 


\section{Abstract}

The interaction of the lantibiotic gallidermin and the glycopeptide antibiotic vancomycin with bacterial membranes was simulated using mass sensitive biosensors and isothermal titration calorimetry (ITC). Both peptides interfere with cell wall biosynthesis by targeting the cell wall precursor lipid II, but differ clearly in their antibiotic activity against individual bacterial strains. We determined binding affinities of vancomycin and gallidermin to model membranes \pm lipid II in detail. Both peptides bind to DOPC/lipid II membranes with high affinity $\left(\mathrm{K}_{\mathrm{D}} 0.30 \mu \mathrm{M}\right.$ and $\left.0.27 \mu \mathrm{M}\right)$. Gallidermin displayed also strong affinity to pure DOPC membranes $(0.53 \mu \mathrm{M})$ an effect that was supported by ITC measurements. A surface acoustic wave (SAW) sensor allowed measurements in the picomolar concentration range and revealed that gallidermin targets lipid II at an equimolar ratio and simultaneously inserts into the bilayer. These results indicate that gallidermin, in contrast to vancomycin, combines cell wall inhibiton and interference with the bacterial membrane integrity for potent antimicrobial activity.

Keywords: Antibiotics; Gallidermin; Isothermal Titration Calorimetry (ITC); Lipid II; Surface Acoustic Wave (SAW) biosensor; Vancomycin;

\section{Abbreviations:}

AMPs, antimicrobial peptides; DOPC, 1,2-dioleoyl-sn-glycero-3-phosphocholine; DOPG, 1,2-dioleoyl-sn-glycero-3-phosphoglycerol; ITC, Isothermal Titration Calorimetry; MC 540, merocyanine 540; MIC, minimal inhibitory concentration; QCM, Quartz Crystal Microbalance; SAW, Surface Acoustic Wave sensor; 


\section{Introduction}

Several antibiotics display their activity by interacting with the bacterial membrane. Beside non-specific membrane disturbing activities, as described for many antimicrobial peptides (AMPs) [1,2], target related modes of action are known. To date several antibiotic compounds were described, which bind to lipid anchored cell wall precursors, and thus inhibit cell wall biosynthesis. The first described example was vancomycin, a glycopeptide that targets a key molecule of peptidoglycan biosynthesis, the cell wall precursor lipid II [3-5]. Later, several lantibiotics, lanthionine containing peptides, were identified, which also target lipid II. In the case of nisin, the prototype of the type-A lantibiotics, the interaction with lipid II leads to a dual mode of action, i.e. cell wall biosynthesis is blocked, and in addition, lipid II is recruited as a pore constituent for permeabilizing the bacterial membrane [6-10].

Gallidermin, a type-A lantibiotic produced by Staphylococcus gallinarum shows striking structural similarities to nisin in the N-terminal double ring system which had been identified as the binding motif for targeting the pyrophosphate moiety of lipid II [11]. In contrast to nisin, due to the short molecular length, gallidermin is hardly able to form pores in membranes via peptide-lipid II complexes. Therefore, the primary mode of action of gallidermin is obviously based on the interaction with lipid II resulting in inhibition of cell wall synthesis [12].

Nevertheless, although gallidermin and vancomycin target the same molecule in the bacterial membrane, striking differences in the antibiotic activity against some bacterial strains are observed. While the MICs of gallidermin and vancomycin against Staphylococcus simulans 22 are in the $\mu \mathrm{M}$ range, $0.15 \mu \mathrm{M}$ and $0.05 \mu \mathrm{M}$, respectively, gallidermin is much more potent than vancomycin against e.g. Lactobacillus lactis HP and Lactobacillus fermentum ATCC $9338(0.005 \mu \mathrm{M}$ and $0.001 \mu \mathrm{M}$ vs. $0.21 \mu \mathrm{M}$ and $>6.9 \mu \mathrm{M})$ 
(unpublished). These observation might be explained by the different membrane interactions of both peptides. Vancomycin targets the D-Ala-D-Ala motif of lipid II [13,14] and has not been described to interact with bacterial membranes, which is in good agreement with the hydrophilic polar structure of the glycopeptide (Figure 1). In contrast, gallidermin comprises hydrophobic moieties which allow for membrane interaction. This is in line with our recent results showing that gallidermin strongly associates with model membranes [15]. It might be anticipated that insertion of gallidermin into the bilayer has an impact on membrane order, and thus indirectly affects the vital function of lipid II and contributes to antimicrobial activity.

Here we compared gallidermin and vancomycin with respect to the membrane interaction and targeting of lipid II to get a deeper insight into gallidermin activities. The use of model membranes in combination with different analytical techniques allowed us to focus on peptide-membrane-interactions, as schematically depicted in Figure 1.

Cyclic voltammetry (data not presented) could exclude that both peptides affected the membrane barrier function. Kinetic binding constants of both peptides to DOPC \pm lipid II model membranes were determined using a quartz crystal microbalance (QCM) and data from a surface acoustic wave sensor (SAW) allowed further insight into gallidermin membrane interactions at varying peptide/lipid ratios. These findings were further supported by isothermal titration calorimetry (ITC) to provide the thermodynamic profile of peptide-membrane interaction $[16,17]$. ITC has been successfully applied in a number of studies to analyze the membrane interaction of different peptides and proteins [18-21]. However, to the best of our knowledge, ITC has neither been used to analyze the interaction of peptides with lipid II containing membranes nor gallidermin in general.

Our data indicate that gallidermin, in contrast to vancomycin, interacts strongly with model membranes and simultaneously targets lipid II nearly at a 1:1 binding ratio. This sheds 
light on the mode of action of gallidermin; especially on the processes at the bacterial membrane surface which result in effective killing.

\section{Materials and Methods}

\subsection{Materials}

1-hexadecanethiol $\left(\mathrm{C}_{16} \mathrm{H}_{33} \mathrm{SH}\right)$ was purchased from FLUKA (Neu-Ulm, Germany). 1,2dioleoyl-sn-glycero-3-phosphocholine $\quad$ (DOPC), 1,2-dioleoyl-sn-glycero-3phosphoglycerol (DOPG), 11-amino-1-undecanethiol hydrochloride, merocyanine 540 (MC 540), and vancomycin were purchased from Sigma-Aldrich (München, Germany). Gallidermin was purchased from Genmedics (Reutlingen, Germany), and C55P was from Larodan (Malmö, Sweden). Lipid II was synthesized using membrane preparations of $M$. luteus DSM 1790 [22] and purified as described [23].

Millipore $^{\circledR}$ water was taken from water Purification System Elix 5 (Millipore GmbH, Schwalbach, Germany). ITC buffers were prepared from $25 \mathrm{mM}$ Tris, and $50 \mathrm{mM}$ sodium chloride (Sigma Aldrich, München, Germany) at $\mathrm{pH}=7.4$. PBS $(\mathrm{pH}=7.4$ ) was prepared according to standard protocols.

\subsection{QCM experiments}

The modification of the quartz crystals has already been described [24,25]. A DOPC or $\mathrm{DOPG} \pm 0.1 \mathrm{~mol} \%$ lipid II layer was transformed by Langmuir-Blodgett technique onto a 1-hexadecanethiol covered quartz crystal. QCM experiments were performed at $25^{\circ} \mathrm{C}$ using a LiquiLab21 system (ifak e.V., Barleben, Germany). After reaching a constant frequency, $3 \mathrm{~mL}$ of a $1 \mu \mathrm{M}$ peptide solution (gallidermin, and vancomycin, respectively) were injected into the flow system. The run was stopped after reaching a constant frequency again. The binding constants were calculated from the frequency run as described [15]. 


\subsection{Liposome preparation}

Liposomes were prepared using the lipid hydration technique. First, a $30 \mathrm{mM}$ aliquot of DOPC, dissolved in chloroform, was put in a $10 \mathrm{~mL}$ round-bottom flask. Chloroform was removed under vacuum by using a rotary evaporator resulting in a thin lipid film. Small glass pellets and $5 \mathrm{~mL}$ of Millipore ${ }^{\circledR}$ water or the indicated buffers were added to the batch and vortexed. Spontaneously formed multilamellar vesicles were shaken overnight and extruded using a Lipex Basic Extruder (Northern Lipids, Vancouver, Canada) seven times through an Isopore ${ }^{\circledR}$ polycarbonate filter with a pore size of $0.1 \mu \mathrm{m}$ (Millipore $\mathrm{GmbH}$, Schwalbach, Germany) resulting in large unilamellar vesicles (LUVs). Liposomal particle size was characterized by dynamic light scattering (Malvern autosizer IIc, Malvern, UK). Phospholipid concentration was calculated according to the inorganic phosphate determination using ascorbate-molybdate mixture and colorimetric detection of the resulting dye at $812 \mathrm{~nm}$, as described by Ames and Dubin [26].

\subsection{SAW measurements}

The S-Sens ${ }^{\circledR}$ K 5 surface acoustic wave biosensor (SAW GmbH, Bonn, Germany) was applied with the two frequency measurement mode between $144 \mathrm{MHz}$ and $152 \mathrm{MHz}$. The gold surface of the $\mathrm{SiO}_{2}$ sensor chips was functionalized by self-assembled monolayers of 11-amino-1-undecanethiol hydrochloride $(2 \mathrm{mM})$ overnight. After washing with ethanol and Millipore ${ }^{\circledR}$ water, the sensor chips were placed into the chip reader unit of the SAW device and the frequency optimum was determined. The measurement temperature was $25^{\circ} \mathrm{C}$ and the flow rate was set to $40 \mu \mathrm{L} / \mathrm{min}$. After an equilibration time of $10 \mathrm{~min}, 300 \mu \mathrm{L}$ of liposome solution $(1 \mathrm{mM})$ were added. Subsequent peptide injections of $150 \mu \mathrm{L}$ were performed in a concentration range from $10^{-13} \mathrm{M}$ to $10^{-6} \mathrm{M}$. Changes in phase shift and amplitude were registered online. 


\subsection{ITC measurements}

All measurements were carried out with a Microcal VP-ITC calorimeter (Microcal, Northampton, USA). The VP-ITC device was electrically calibrated. All samples were degassed and temperated before measurement. $25^{\circ} \mathrm{C}$ was chosen as the experimental temperature. The reference power was set to $8 \mu \mathrm{cal} / \mathrm{sec}$, using a syringe stirring speed of $350 \mathrm{rpm}$. Titrations were performed by a number of 50 injections of each $5 \mu \mathrm{L}$ steps of LUV aliquots $(30 \mathrm{mM})$ into the sample cell containing $1.43 \mathrm{~mL}$ peptide at a concentration of $100 \mu \mathrm{M}$. The change in heat rate during the titration steps was registered in real time. Raw data were processed using the Origin ${ }^{\circledR}$ software provided with the instrument. Blank values were determined in control titrations by injecting LUVs into the indicated solvents. Although dilution heat was small and negligible, it was included in the final analysis. Under assumption of an one-site binding model with the ligand in the sample cell, thermodynamic parameters as enthalpy $(\Delta \mathrm{H})$, entropy $(\Delta \mathrm{S})$ and the equilibrium binding constant $\left(\mathrm{K}_{\mathrm{D}}\right)$ were calculated.

\subsection{Merocyanine 540 assay}

Merocyanine 540 (MC540) was added to the liposome batch from a stock solution (20 $\mu \mathrm{g} / \mathrm{mL}$ PBS) to reach a final dye:lipid molar ratio of 1:100. MC 540 containing liposomes were incubated for $20 \mathrm{~min}$ at $8^{\circ} \mathrm{C}$ before peptides were added in a peptide:lipid ratio of 1:10 or 1:1, resp. The fluorescence was measured with a spectrofluorometer (Perkin Elmer LS1, Waltham, USA) at $584 \mathrm{~nm}$ and excitation wavelength at $540 \mathrm{~nm}$.

\subsection{Statistical methods}

QCM experiments were repeated for at least four times. Kinetic binding constants are represented as mean value \pm standard deviation. The statistical significance of the effects 
was determined by the unpaired Student`s t-test. A p value of $\leq 0.05$ was considered for statistical significance. ITC experiments and SAW measurements were repeated for at least seven times, representative ITC scans and SAW curves were selected for presentation. The fluorescence measurements were repeated three times.

\section{Results and Discussion}

\subsection{Kinetics of peptide binding to model membranes}

Kinetic constants of peptide binding to model membranes give an insight into antibiotic activity referring to different modes of membrane interaction or entering the bacterial cell. A QCM biosensor was applied to detect peptide binding kinetics of gallidermin and vancomycin. To consider the role of lipid II as molecular target and its impact on binding intensity, $0.1 \mathrm{~mol} \%$ lipid II was added to the matrix lipids. In accordance to other peptide/model membrane studies [27-33] and to avoid non-specific binding of the cationic antimicrobial peptides to the membrane surface, DOPC was initially selected as membrane lipid. As depicted in Fig 2A, lipid II as the bacterial target is crucial for vancomycin membrane binding. Compared to pure DOPC membranes, vancomycin binding to DOPC/lipid II is nearly tenfold stronger $(0.30 \mu \mathrm{M}$ vs. $2.42 \mu \mathrm{M})$. Gallidermin displays a comparable high affinity binding in presence of lipid II, but a much higher affinity to pure DOPC membranes $(0.53 \mu \mathrm{M})$ than vancomycin. This suggests to differences in the mode of membrane interaction of both peptides, independent of lipid II targeting. While vancomycin membrane interaction have not been reported [34,35], the gallidermin structure (Fig. 1) give clear indication for membrane interaction forced by the hydrophobic moieties.

With respect to the net negative charge of bacterial membranes, the twofold positive charge of gallidermin should also have an impact on the non-targeted membrane affinity. 
In order to simulate this, gallidermin binding affinies to DOPC/DOPG (9:1) membranes were detected (Fig. 2B). Membrane charge, which is assumed to induce the inital electrostatic attraction of the peptide to the membrane, is of minor effect in this approach. Although the affinities of gallidermin to the charged membranes are slightly higher than to the neutral ones, the differences between pure and lipid II containing membranes in turn are marginal $(0.22 \mu \mathrm{M}$ vs. $0.11 \mu \mathrm{M}$, resp.). This indicates that membrane charge only plays a minor role in membrane affinity of gallidermin.

To evaluate the binding process of both peptides to the DOPC membranes in more detail, association and dissociation rate of vancomycin were determined as depicted in Fig. 2C and $\mathrm{D}$ in comparison to the recently published gallidermin binding data [15]. Lipid II induces a faster association rate of vancomycin (Fig. 2C) and a decelerated dissociation (Fig. 2D) than on pure DOPC. In contrast to vancomycin, gallidermin binding is differently affected by lipid II. Gallidermin has more than a twofold higher association rate to pure DOPC membranes than in presence of lipid II, which is threefold higher than for vancomycin (Fig. 2C). This dominantly contributes to the high binding affinity to the DOPC membranes $(0.53 \mu \mathrm{M})$ (Fig. $2 \mathrm{~A})$, since dissociation rates of both peptides are comparable, indicating the target role of lipid II (Fig. 2D).

Although we have to consider critically that the QCM measurements are based on a fixed peptide/lipid ratio and on an asymmetric supported model membrane of a covalently fixed first monolayer, which might have an impact on lipid II membrane insertion and accessibility, the high association of gallidermin indicates a certain tendency for membrane insertion. Changes in membrane permeability by both peptides or the ability to form pores into DOPC membranes were excluded by cyclic voltammetry measurements (data not shown). 


\subsection{Detecting different modes of gallidermin membrane binding}

To further evaluate the mechanisms behind the high binding affinity of gallidermin to pure DOPC membranes and the minor effects of lipid II in increasing the binding, a SAW biosensor technique was applied. This technology is based on the changes of phase and amplitude of surface acoustic waves due to binding processes of e.g. peptides on the surface of a sensor chip, and further allows distinguishing signals from mass changes and viscoelastic properties. The number of SAW binding studies is limited and only one recent study focused on the application of SAW in the field of antimicrobial peptide binding to model membranes [36].

The supported membranes were created by spreading of liposomes at the sensors. In contrast to the fixed peptide/lipid ratio in the QCM experiments, step like increasing concentrations of gallidermin were allowed to interact with the membrane, starting with $0.1 \mathrm{pM}$. The phase changes, indicating the interaction of gallidermin with pure DOPC (Fig. 3A, upper line) displayed two minor signals at $0.1 \mathrm{pM}$ and $1 \mathrm{pM}$ followed by a very strong phase shift at $10 \mathrm{pM}$ concentration (dashed line). The associated changes in amplitude (Figure 3B, upper line) indicate the viscoelasticity of the surface bound structures. The increase of amplitude at $10 \mathrm{pM}$ gallidermin (dashed line) can clearly be addressed to a membrane rigidification and suggests a gallidermin insertion into the membrane. This supports the assumption that gallidermin tends to insert into the membrane, which occurs mainly unique at a certain threshold concentration, obviously leading to a membrane saturation. Vancomycin displayed no interaction under identical conditions (not shown).

Considering the lipid II containing membrane, gallidermin revealed a different mode of interaction compared to the pure DOPC. Small signals in phase shift (Fig. 3A, lower line) indicate weak binding events at $1 \mathrm{pM}$ and $10 \mathrm{pM}$ and were followed by a stronger and reversible signal at $100 \mathrm{pM}$ gallidermin. These signals indicate the binding of gallidermin to lipid II. To further evaluate these findings, the most prominent phase shift at $100 \mathrm{pM}$ 
gallidermin (dotted line) was analyzed with respect to the peptide/lipid II ratio. The number of lipid II molecules ( $0.1 \mathrm{~mol} \%$ in the lipid matrix $)$ at the sensor surface was calculated, dividing the sensitive surface by the mean phospholipid head group size, to be about $1.4 \times 10^{10}$ lipid II molecules. This excellently fits with the peak at $100 \mathrm{pM}$ gallidermin, which corresponds to about $1.1 \times 10^{10}$ gallidermin molecules in the measurement compartment. Thus, the lipid II recognition of gallidermin is confirmed and occured at a binding ratio of roughly $1: 1$. Further insight is given by the course of amplitude of this measurement (Fig. 3B, lower line). The binding of gallidermin to lipid II at the three lowest concentrations $(0.1-10 \mathrm{pM})$ is associated with small drops in amplitude, indicating an increase in viscoelasticity. This is also true for the dominat binding at $100 \mathrm{pM}$ (dotted line), but after an initial drop, the amplitude increases evidently. This refers to the fact that lipid II binding of gallidermin is superimposed by membrane insertion of the peptide. Interestingly, the concentration range of the gallidermin lipid II binding signals in the SAW measurements $(0.1 \mathrm{nM}$ gallidermin) is in the range of the in vivo activity of gallidermin against sensitive strains, determined as MICs; i.e. Lactobacillus lactis HP and Lactobacillus fermentum ATCC 9338, $5 \mathrm{nM}$ and $1 \mathrm{nM}$, respectively.

Summarizing, the SAW measurements give clear indications for a lipid II recognition of gallidermin and dominant binding at roughly equimolar peptide/lipid II ratio and simultaneous membrane insertion of gallidermin. The reduction of gallidermin membrane insertion in presence of lipid II is obviously caused by membrane stabilizing effects of lipid II, which we have recently illustrated microscopically by reduced lipid lateral diffusion rates in presence of lipid II [25].

\subsection{Peptide interaction with pure DOPC membranes detected by ITC}

The present data indicate strong differences of gallidermin and vancomycin in the mode of membrane interaction, although both peptides share the targeting of lipid II as basis for 
antibiotic activity. To further evaluate the mode of membrane interaction of both peptides, ITC as promising thermoanalytical technique was applied here as already described for other peptide membrane interactions, such as penetratin [18] or magainin [19]. A recently published ITC study of vancomycin binding to synthetic fragments of the cell wall peptidoglycans confirmed a 1:1 binding stochiometry between vancomycin and the D-AlaD-Ala moiety [20]. Breukink et al. investigated the influence of membrane charge on the binding of nisin by ITC [21]. Since no reference data exist on the membrane interactions of gallidermin, the experimental parameters were selected according to former ITC investigations of peptide-lipid interactions by Seelig et al. [37]. Referring to these established experiments and the fact that the higher concentrated reactant has to be injected into the lower concentrated one, DOPC liposomes were titrated into the peptide solution. At each titration step, $150 \mathrm{nmol}$ DOPC liposomes were added to an initial amount of 143 nmol peptide. Every further injection increased the molar ratio of lipid:peptide by the value of approximately 1. Although this sequence of peptide membrane contacts is not in accordance to the conditions of the biosensor measurements before, the resulting ITC scan should represent a comprehensive image to interpret the type of interactions. With respect to the high concentrations of peptide used, crystallization effects could be excluded considering the sufficient solubility of the peptide in aqueous solution $(2 \mathrm{mg} / \mathrm{mL})$.

Gallidermin titration with DOPC vesicles displayed a typical sigmoidal ITC trace of exothermic peaks (Fig. 4A). The heat rate decreased during the titration until reaching constant values, indicating a saturation of peptide-membrane interaction. In contrast to gallidermin, almost no changes in the heat rate of vancomycin titration with DOPC vesicles were detectable indicating only minor interactions (Fig. 4B). This is also reflected in about tenfold lower enthalpic changes of vancomycin compared to gallidermin $(-508 \pm$ $165 \mathrm{cal} / \mathrm{mol}$ vs. $-5455 \pm 391 \mathrm{cal} / \mathrm{mol})$. 
These differences reflect the biosensor binding studies and refer that the exothermic peaks represent peptide membrane interaction. The trace of titration peaks of gallidermin allow for the calculation of a binding affinity of $3.28 \mu \mathrm{M}$, which is lower, but in a comparable range of the QCM data $(0.53 \mu \mathrm{M})$. The differences might be explained by the experimental parameters, such as peptide/lipid ratio, or planar vs. liposomal model membranes.

To exclude that these findings were related to $\mathrm{pH}$ effects, the ITC measurements were performed under indicated buffer conditions $(\mathrm{pH}$ 7.4). The results confirmed that gallidermin displayed a pronounced interaction with the model membranes, while vancomycin in turn showed no membrane interaction; not even at higher temperatures.

To further focus on the mode of peptide membrane interaction, a fluorescence assay on the basis of merocyanine 540 was applied. MC 540 interacts with membranes changing its spectral characteristics according to the membrane fluidity. An increase in membrane fluidity corresponds with higher fluorescence emission at $584 \mathrm{~nm} \mathrm{[38].} \mathrm{Peptide} \mathrm{insertion}$ into the liquid expanded DOPC bilayer should induce a condensing effect on the membrane order. Consequently, a resulting rigidification of the liposomes should be followed by a fluorescence decrease.

In accordance to the ITC experiments, gallidermin was added to MC540-pretreated DOPC liposomes at a molar ratio of 1:10 (representing the tenth titration peak), which induced a fluorescence decrease by $43 \%$ (reduction from 282 to 160 fluorescence units). In contrast, similar amounts of vancomycin had no influence on the fluorescence (248 to $259 \mathrm{u}$, corresponding to an increase of $4 \%$ ). Further increase of gallidermin concentration to equimolar ratio reduced the emission by $55 \%$ (128 u), while vancomycin has only a minor effect at this concentration $(187 \mathrm{u})$. These data strongly support the assumption derived from the SAW amplitude measurements (Fig. 3B, upper line) that gallidermin inserts into the membrane causing a rigidification, which is not true for vancomycin. 


\subsection{ITC investigations on peptide interaction with DOPC/lipid II membranes}

To investigate, whether the targeting of lipid II has an impact on the ITC traces and thus to obtain more interpretation of the scans in Figure 4, experiments were continued titrating gallidermin and vancomycin with DOPC liposomes containing $0.1 \mathrm{~mol} \%$ lipid II (Fig. 5). The exothermic trace of gallidermin (Fig. 5A) displayed a slight change in the curve shape compared to pure DOPC (Fig. 4A) suggesting that a saturation of the reaction occurred at lower concentrations. The changes in the heat rate were slightly diminished although the blank values were higher. Similar data could be obtained considering the influence of buffer or changed temperature in the ITC experiments (not shown). Lipid II has a certain impact on gallidermin binding, but it is not evident whether these changes represent direct lipid II binding of gallidermin, or membrane changes induced by lipid II.

Higher blank levels were also evident in the vancomycin titration trace (Fig. 5B). The change in enthalpy data (gallidermin $-5455 \mathrm{cal} / \mathrm{mol}$ vs. $-3018 \mathrm{cal} / \mathrm{mol}$; vancomycin -508 cal/mol vs. $-1353 \mathrm{cal} / \mathrm{mol}$ ) are also not indicative for lipid II recognition of both peptides. However, this does not exclude an interaction between vancomycin and lipid II, but demonstrate that in this case, the ITC method is not sensitive enough to detected the targetmediated binding of vancomycin and gallidermin. Furthermore, this finding is no contradiction to the data of Rekharsky et al. [20] who detected the interaction of vancomycin with much higher concentrations of D-Ala-D-Ala, compared to lipid II concentration in model membranes in our study.

To confirm that the minor changes in the ITC scans in presence of lipid II were induced rather by indirect effects of the lipid II isoprenoid chain than by target recognition of the lipid II head group, we incorporated the lipid II membrane anchor C55P into the DOPC liposomes. Since the peptide side chain, the sugar moiety, and the second phosphate are missing, a specific recognition by gallidermin or vancomycin can be excluded. Gallidermin 
displayed a binding affinity of $0.21 \mu \mathrm{M} \pm 0.07 \mu \mathrm{M}$ to DOPC/C55P membranes in QCM experiments. This is comparable to gallidermin affinity to both, pure and lipid II containing DOPC membranes, which indicates no specific effects of C55P.

The gallidermin trace in presence of $\mathrm{C55P}$ (Figure 6) is comparable to the lipid II scan, the higher exothermic peaks (titration steps 10-18) imply a slightly stronger interaction, most likely incorporation of gallidermin into the membrane than in presence of lipid II. The ITC trace of vancomycin is identical to pure DOPC indicating no membrane interaction. Therefore, these data suggest that the curve characteristics in Figure 5 are mainly induced by membrane effects in the hydrophobic moiety. Recognition of lipid II by both peptides can not be detected by ITC.

To further differentiate the effects of lipid II and C55P, we calculated the molar amount of membrane interacting gallidermin $X_{p}$ for each injection $\mathrm{k}$ according to Eq. 1, which is described in detail in $[19,37]$

$$
X_{p}^{(k)}=n_{p}^{(k)} / n_{p}=\sum_{1}^{k} \Delta h_{k} / \sum_{1}^{n} \Delta h_{k}
$$

Plotting the molar amount of membrane interacting gallidermin $X_{p}$ against the first 25 titration steps (Fig. 7) illustrates that lipid II and C55P reduce the gallidermin interaction with DOPC membranes to a similar extent. This suggests to a dominant effect of the isoprenoid chain of lipid II and C55P on the membrane and thus, on gallidermin membrane insertion. This finding totally reflects the SAW binding data that gallidermin membrane insertion occurs at higher concentrations and to a lower degree, when lipid II is present. Taking the slope from step 10 to 20 as an indicator for membrane interaction, $(0.0343$; 0.0285; and 0.0181 for DOPC, DOPC/C55P, DOPC/lipid II, respectively), the calculated affinities from the titration traces of gallidermin are in good agreement $(3.28 \mu \mathrm{M} ; 7.09$ $\mu \mathrm{M}$; and $18.9 \mu \mathrm{M})$. The slightly lower slope in the presence of lipid II compared to C55P can reflect the recognition of lipid II interfering with unspecific membrane internalization. 
These data also allow for the interpretation that membrane inserted gallidermin disturb membrane integrity. In addition to the "classical" targeting of lipid II at the membrane surface, these inner membrane effects might also add to the sequestration of lipid II from the sites of cell wall biosynthesis and thus contribute to the antibiotic effects of gallidermin [39].

\section{Conclusions}

Overall, the data presented here provide evidence for non-targeted membrane activities of gallidermin in synergy with lipid II mediated interactions leading to cell wall inhibition in combination with membrane disturbing effects. Both activities will be affected by the bacterial membrane characteristics, which might therefore be key for the activity against individual strains. In the case of vancomycin that does not interact with the membrane, other factors e.g. accessibility of lipid II in the bacterial membrane are the main determinants of antimicrobial activity. The model membrane approaches used here are powerful tools to analyze membrane activities of antimicrobial peptides. Especially the SAW technique is a sensitive method to detect peptide-target interactions.

\section{Acknowledgements}

This work was supported by the Deutsche Forschungsgemeinschaft, BE2242/2-1, FOR 854, and GRK 677. The authors are thankful to Prof. Joachim Seelig for helpful discussions concerning the ITC data, Dr. Thomas Gronewold for help in interpretation of the SAW data, and Prof. Hans-Georg Sahl for providing the lipid II. We thank H. Falkenstein-Paul for excellent technical assistance, and M. Josten for mass determination of the peptides. 


\section{References}

[1] Y. Shai, Mode of action of membrane active antimicrobial peptides, Biopolymers. 66 (2002) 236-248.

[2] K. Lohner, S.E. Blondelle, Molecular mechanisms of membrane perturbation by antimicrobial peptides and the use of biophysical studies in the design of novel peptide antibiotics, Comb. Chem. High Throughput Screen. 8 (2005) 241-256.

[3] A.N. Chatterjee, H.R. Perkins, Compounds formed between nucleotides related to the biosynthesis of bacterial cell wall and vancomycin, Biochem. Biophys. Res. Commun. 24 (1966) 489-494.

[4] K.K. Wong, D.L. Pompliano, Peptidoglycan biosynthesis. Unexploited antibacterial targets within a familiar pathway, Adv. Exp. Med. Biol. 456 (1998) 197-217.

[5] E. Breukink, B. de Kruijff, Lipid II as a target for antibiotics, Nat Rev Drug Discov. 5 (2006) 321-332.

[6] E. Breukink, H.E. van Heusden, P.J. Vollmerhaus, E. Swiezewska, L. Brunner, S. Walker, et al., Lipid II is an intrinsic component of the pore induced by nisin in bacterial membranes, J. Biol. Chem. 278 (2003) 19898-19903.

[7] H. Brötz, M. Josten, I. Wiedemann, U. Schneider, F. Götz, G. Bierbaum, et al., Role of lipid-bound peptidoglycan precursors in the formation of pores by nisin, epidermin and other lantibiotics, Mol. Microbiol. 30 (1998) 317-327.

[8] H.E. Hasper, B. de Kruijff, E. Breukink, Assembly and stability of nisin-lipid II pores, Biochemistry. 43 (2004) 11567-11575.

[9] S.D. Hsu, E. Breukink, E. Tischenko, M.A.G. Lutters, B. de Kruijff, R. Kaptein, et al., The nisin-lipid II complex reveals a pyrophosphate cage that provides a blueprint for novel antibiotics, Nat. Struct. Mol. Biol. 11 (2004) 963-967.

[10] H.E. van Heusden, B. de Kruijff, E. Breukink, Lipid II induces a transmembrane orientation of the pore-forming peptide lantibiotic nisin, Biochemistry. 41 (2002) 12171-12178.

[11] R. Kellner, G. Jung, T. Hörner, H. Zähner, N. Schnell, K.D. Entian, et al., Gallidermin: a new lanthionine-containing polypeptide antibiotic, Eur. J. Biochem. 177 (1988) 53-59.

[12] R.R. Bonelli, T. Schneider, H. Sahl, I. Wiedemann, Insights into in vivo activities of lantibiotics from gallidermin and epidermin mode-of-action studies, Antimicrob. Agents Chemother. 50 (2006) 1449-1457.

[13] G.M. Sheldrick, P.G. Jones, O. Kennard, D.H. Williams, G.A. Smith, Structure of vancomycin and its complex with acetyl-D-alanyl-D-alanine, Nature. 271 (1978) 223225.

[14] Y. Gilbert, M. Deghorain, L. Wang, B. Xu, P.D. Pollheimer, H.J. Gruber, et al., Single-molecule force spectroscopy and imaging of the vancomycin/D-Ala-D-Ala interaction, Nano Lett. 7 (2007) 796-801.

[15] K. Christ, S. Al-Kaddah, I. Wiedemann, B. Rattay, H. Sahl, G. Bendas, Membrane lipids determine the antibiotic activity of the lantibiotic gallidermin, J. Membr. Biol. 226 (2008) 9-16.

[16] A. Cooper, Microcalorimetry of protein-protein interactions, Methods Mol. Biol. 88 (1998) 11-22.

[17] G.A. Holdgate, Making cool drugs hot: isothermal titration calorimetry as a tool to study binding energetics, BioTechniques. 31 (2001) 164-166, 168, 170 passim.

[18] H. Binder, G. Lindblom, Charge-dependent translocation of the Trojan peptide penetratin across lipid membranes, Biophys. J. 85 (2003) 982-995.

[19] M.R. Wenk, J. Seelig, Magainin 2 amide interaction with lipid membranes: calorimetric detection of peptide binding and pore formation, Biochemistry. 37 
(1998) 3909-3916.

[20] M. Rekharsky, D. Hesek, M. Lee, S.O. Meroueh, Y. Inoue, S. Mobashery, Thermodynamics of interactions of vancomycin and synthetic surrogates of bacterial cell wall, J. Am. Chem. Soc. 128 (2006) 7736-7737.

[21] E. Breukink, P. Ganz, B. de Kruijff, J. Seelig, Binding of Nisin Z to bilayer vesicles as determined with isothermal titration calorimetry, Biochemistry. 39 (2000) 1024710254.

[22] H. Brötz, G. Bierbaum, P.E. Reynolds, H.G. Sahl, The lantibiotic mersacidin inhibits peptidoglycan biosynthesis at the level of transglycosylation, Eur. J. Biochem. 246 (1997) 193-199.

[23] T. Schneider, M.M. Senn, B. Berger-Bächi, A. Tossi, H. Sahl, I. Wiedemann, In vitro assembly of a complete, pentaglycine interpeptide bridge containing cell wall precursor (lipid II-Gly5) of Staphylococcus aureus, Mol. Microbiol. 53 (2004) 675685.

[24] K. Christ, H. Rüttinger, M. Höpfner, U. Rothe, G. Bendas, The detection of UVinduced membrane damages by a combination of two biosensor techniques, Photochem. Photobiol. 81 (2005) 1417-1423.

[25] K. Christ, I. Wiedemann, U. Bakowsky, H. Sahl, G. Bendas, The role of lipid II in membrane binding of and pore formation by nisin analyzed by two combined biosensor techniques, Biochim. Biophys. Acta. 1768 (2007) 694-704.

[26] B. Ames, D. Dubin, The role of polyamines in the neutralization of bacteriophage deoxyribonucleic acid, J. Biol. Chem. 235 (1960) 769-775.

[27] B. Martínez, T. Böttiger, T. Schneider, A. Rodríguez, H. Sahl, I. Wiedemann, Specific interaction of the unmodified bacteriocin Lactococcin 972 with the cell wall precursor lipid II, Appl. Environ. Microbiol. 74 (2008) 4666-4670.

[28] C.J. Arnusch, H. Branderhorst, B. de Kruijff, R.M.J. Liskamp, E. Breukink, R.J. Pieters, Enhanced membrane pore formation by multimeric/oligomeric antimicrobial peptides, Biochemistry. 46 (2007) 13437-13442.

[29] I. Wiedemann, T. Böttiger, R.R. Bonelli, T. Schneider, H. Sahl, B. Martínez, Lipid IIbased antimicrobial activity of the lantibiotic plantaricin C, Appl. Environ. Microbiol. 72 (2006) 2809-2814.

[30] E. Ruhr, H.G. Sahl, Mode of action of the peptide antibiotic nisin and influence on the membrane potential of whole cells and on cytoplasmic and artificial membrane vesicles, Antimicrob. Agents Chemother. 27 (1985) 841-845.

[31] T. Böttiger, T. Schneider, B. Martínez, H. Sahl, I. Wiedemann, Influence of $\mathrm{Ca}(2+)$ ions on the activity of lantibiotics containing a mersacidin-like lipid II binding motif, Appl. Environ. Microbiol. 75 (2009) 4427-4434.

[32] N. Ghalit, J.F. Reichwein, H.W. Hilbers, E. Breukink, D.T.S. Rijkers, R.M.J. Liskamp, Synthesis of bicyclic alkene-/alkane-bridged nisin mimics by ring-closing metathesis and their biochemical evaluation as lipid II binders: toward the design of potential novel antibiotics, Chembiochem. 8 (2007) 1540-1554.

[33] L. Ringstad, E. Protopapa, B. Lindholm-Sethson, A. Schmidtchen, A. Nelson, M. Malmsten, An electrochemical study into the interaction between complementderived peptides and DOPC mono- and bilayers, Langmuir. 24 (2008) 208-216.

[34] D.A. Beauregard, D.H. Williams, M.N. Gwynn, D.J. Knowles, Dimerization and membrane anchors in extracellular targeting of vancomycin group antibiotics, Antimicrob. Agents Chemother. 39 (1995) 781-785.

[35] O. Domenech, G. Francius, P.M. Tulkens, F. Van Bambeke, Y. Dufrêne, M. MingeotLeclercq, Interactions of oritavancin, a new lipoglycopeptide derived from vancomycin, with phospholipid bilayers: Effect on membrane permeability and nanoscale lipid membrane organization, Biochim. Biophys. Acta. 1788 (2009) 1832- 
1840.

[36] J. Andrä, A. Böhling, T.M.A. Gronewold, U. Schlecht, M. Perpeet, T. Gutsmann, Surface acoustic wave biosensor as a tool to study the interaction of antimicrobial peptides with phospholipid and lipopolysaccharide model membranes, Langmuir. 24 (2008) 9148-9153.

[37] J. Seelig, Titration calorimetry of lipid-peptide interactions, Biochim. Biophys. Acta. 1331 (1997) 103-116.

[38] P. Williamson, K. Mattocks, R.A. Schlegel, Merocyanine 540, a fluorescent probe sensitive to lipid packing, Biochim. Biophys. Acta. 732 (1983) 387-393.

[39] H.E. Hasper, N.E. Kramer, J.L. Smith, J.D. Hillman, C. Zachariah, O.P. Kuipers, et al., An alternative bactericidal mechanism of action for lantibiotic peptides that target lipid II, Science. 313 (2006) 1636-1637. 


\section{Legends to Figures}

Figure 1: Structure of the bacterial cell wall precursor lipid II and it target moieties for the lantibiotic gallidermin (A) and the glycopeptide vancomycin (B). Hydrophobic amino acids of both peptides are marked in grey, partly induced by ether bonds (dashed amino acids). In the lower part a schematic outline of the model membrane approaches and techniques used in this study are presented.

Figure 2: Detection of the kinetic constants of vancomycin and gallidermin binding to model membranes by QCM biosensor technique. (A) Binding affinity of both peptides to model membranes of DOPC $\pm 0.1 \mathrm{~mol} \%$ lipid II. Vancomycin binding is strongly increased in the presence of lipid II, while gallidermin displays comparable high affinity to both, pure and lipid II containing DOPC membranes. (B) Comparison of gallidermin binding affinity to non-charged $($ DOPC $\pm 0.1 \mathrm{~mol} \%$ lipid II) or negatively charged (DOPC/DOPG 9/1 $\pm 0.1 \mathrm{~mol} \%$ lipid II) model membranes. Membrane charge is of minor effect for gallidermin membrane affinity. (C) Association rate and (D) dissociation rate of vancomycin and gallidermin binding to DOPC \pm $0.1 \mathrm{~mol} \%$ lipid II. While lipid II increases the association rate of vancomycin, gallidermin has a high association tendency to interact with the pure DOPC membrane, which is reduced in presence of lipid II (C). The dissociation rate clearly indicates the role of lipid II as target for both peptides, inducing slower dissciation rates (D).

Figure 3: SAW experiments of gallidermin binding to pure DOPC and DOPC containing $0.1 \mathrm{~mol} \%$ lipid II model membranes, analyzed by the indicated phase shifts (A) and associated changes in amplitude (B). The dominant peak of gallidermin binding to pure DOPC in (A) at a $10 \mathrm{pM}$ concentration (dashed line) is accompanied by an increase in amplitude (B), which can be related to a membrane insertion of gallidermin in the DOPC bilayer and subsequent membrane rigidification. In presence of lipid II, the target recognition is most evident at $100 \mathrm{pM}$ concentration (A; dotted line), roughly corresponding to a $1: 1$ (gallidermin:lipid II) binding ratio.Target recognition is 
superimposed by a membrane insertion of gallidermin, illustrated in the amplitude trace (B, dotted line).

Figure 4: Representative ITC-scans of titrating DOPC liposomes into gallidermin (A) or vancomycin (B). The much higher enthalpic changes of gallidermin vs. vancomycin refer to stronger membrane interactions of gallidermin.

Figure 5: ITC traces of titrating DOPC/0.1 mol\% lipid II into gallidermin (A) and vancomycin (B). The presence of lipid II possesses only marginal effects, the titration traces do not allow to derive targeting effects of both peptides to lipid II.

Figure 6: ITC traces of titrating DOPC/0.1 mol\% C55P into gallidermin (A) and vancomycin (B) to differentiate between target recognition of lipid II and unspecific effects of lipid II isoprenoid chain in the membrane. The comparable behavior of C55P and lipid II (Fig. 5) refers to the effects of the isoprenoid moiety of lipid II on the membrane.

Figure 7: Comparison of gallidermin titration with DOPC, DOPC/0.1 mol\% lipid II or DOPC/0.1 mol\% C55P with respect to the molar amount of membrane interacting gallidermin according to Eq. 1 at the first 25 titration steps. The slope of the curves confirms that gallidermin is stronger internalized into pure DOPC membranes. The presence of lipid II and C55P decreases the rate of membrane insertion similarly indicating the effect of the isoprenoid chain on the membrane structure. 

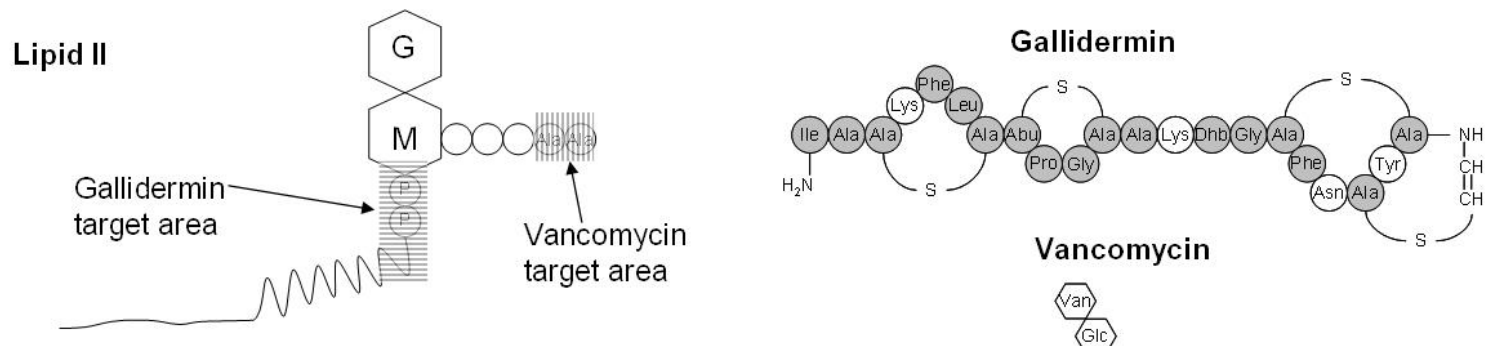

van

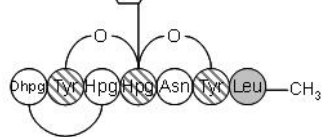

permeability non-targeted membrane interaction

targeted membrane interaction

membrane

insertion

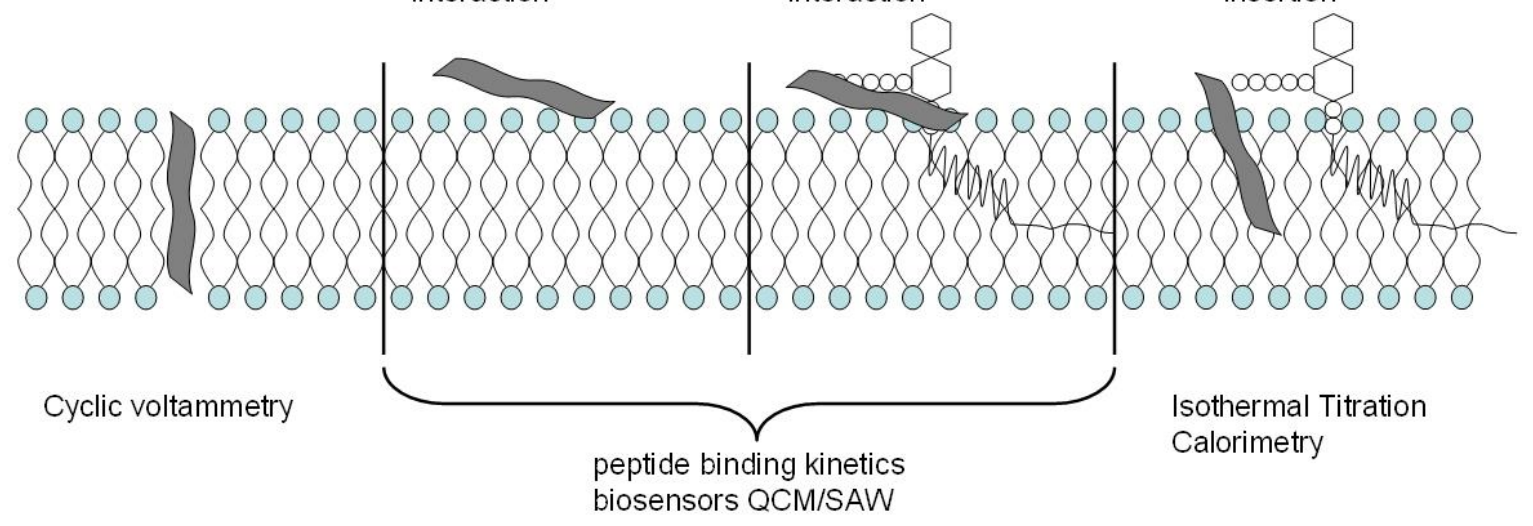

Fig. 1 
Fig. 2
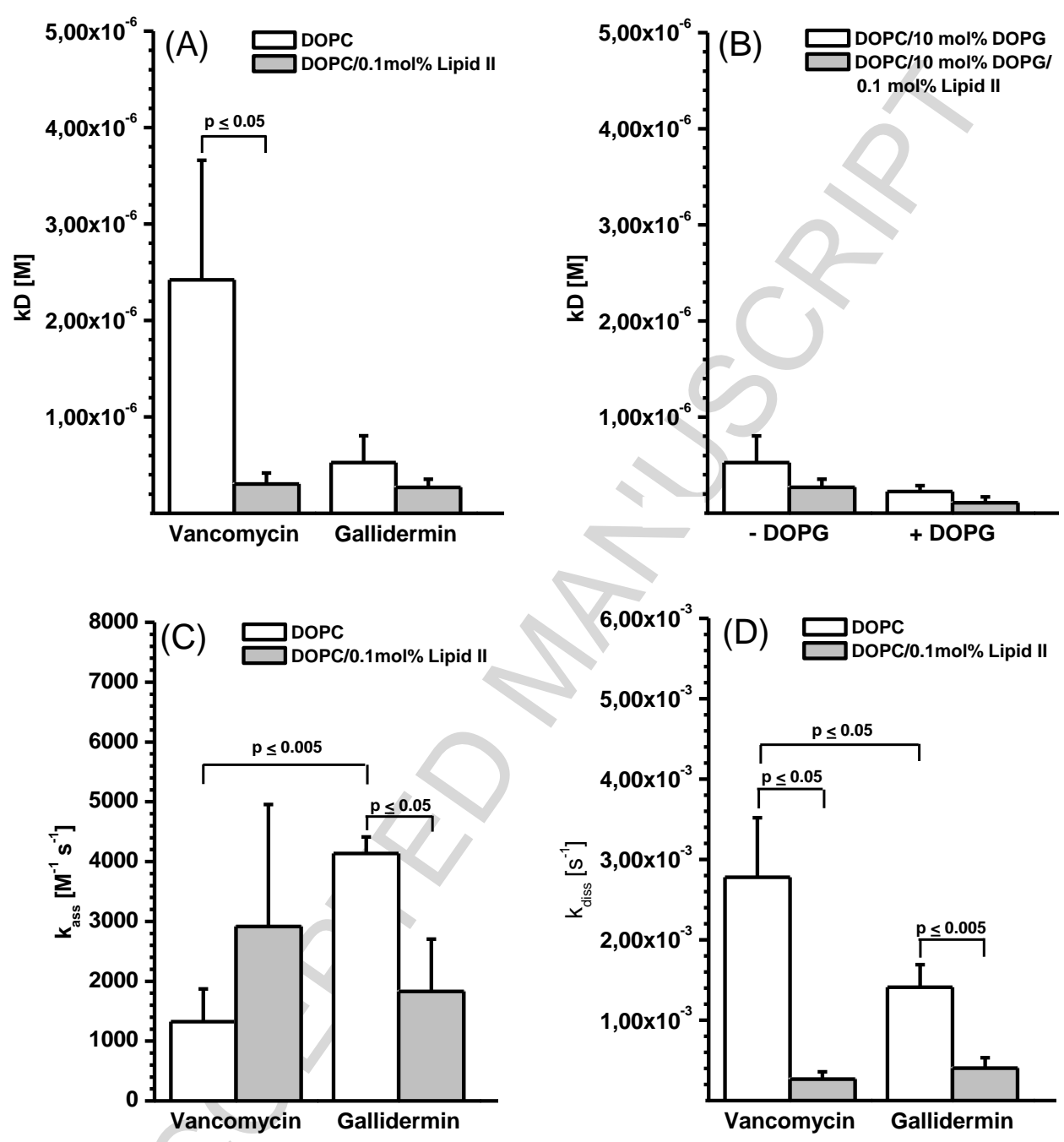


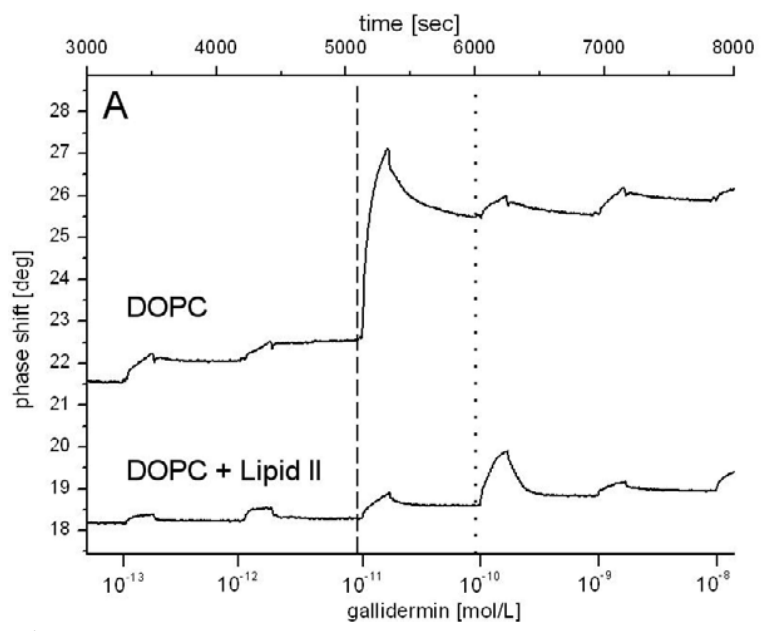

Fig. 3

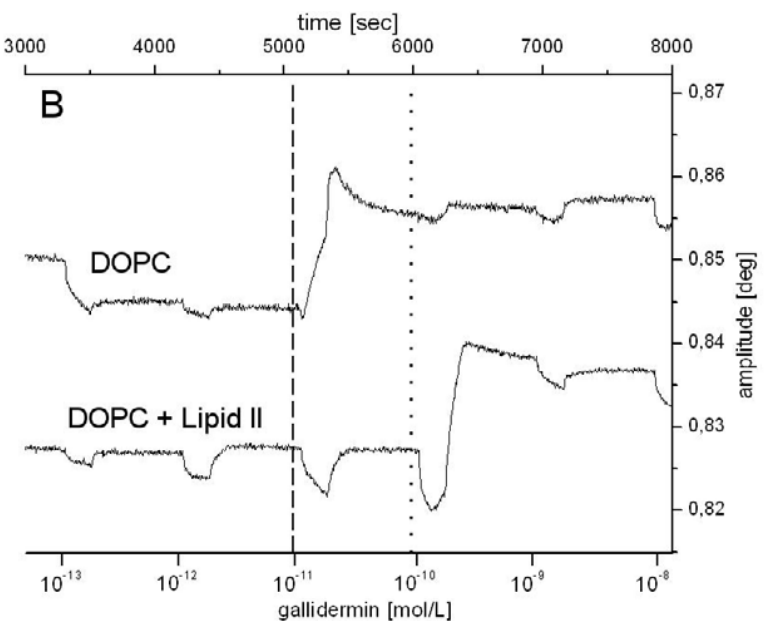


A

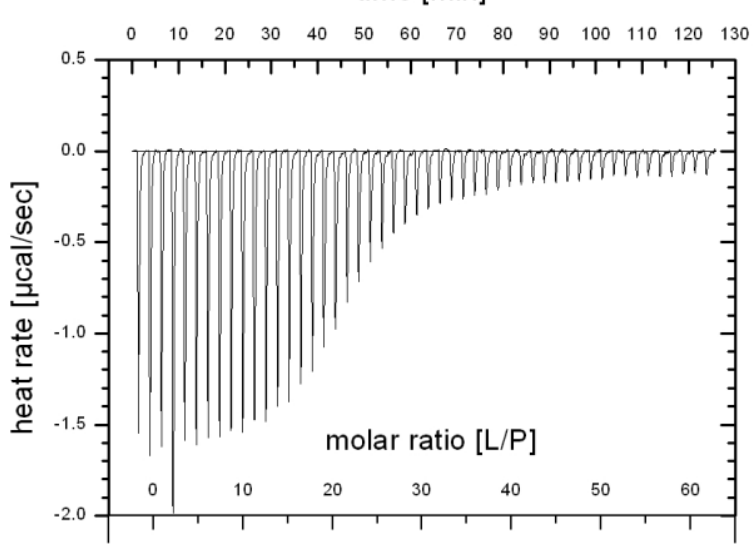

B

time [min]

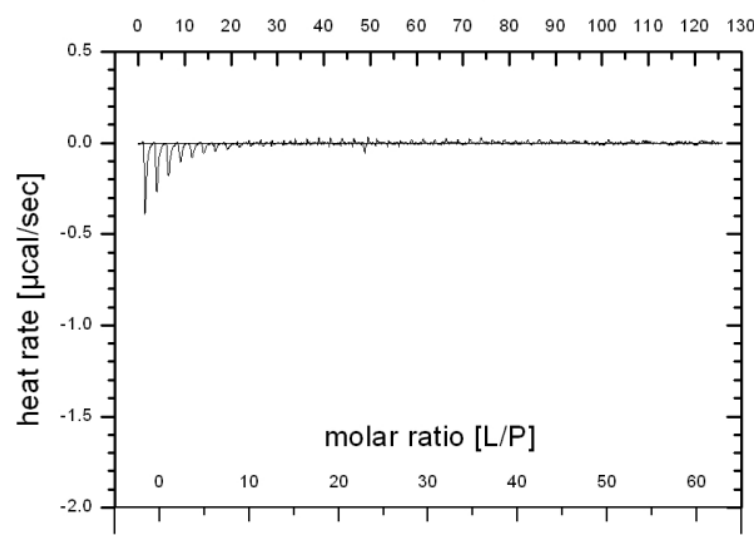

Fig. 4 
A time [min]

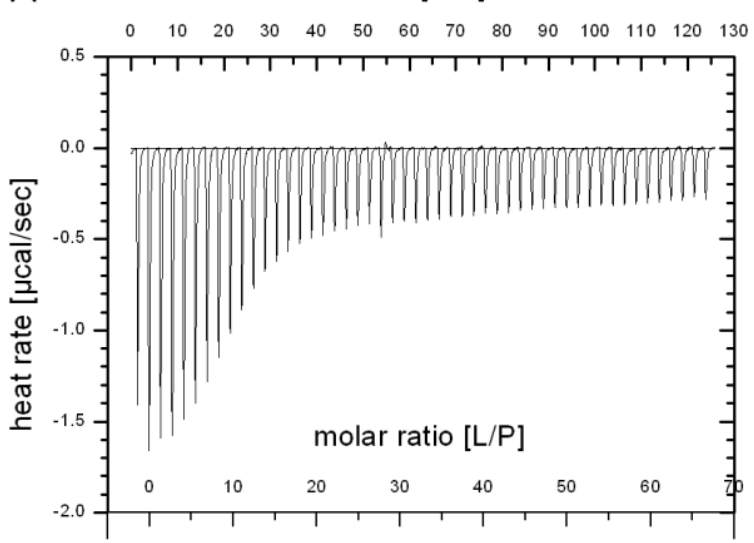

Fig. 5
B time [min]

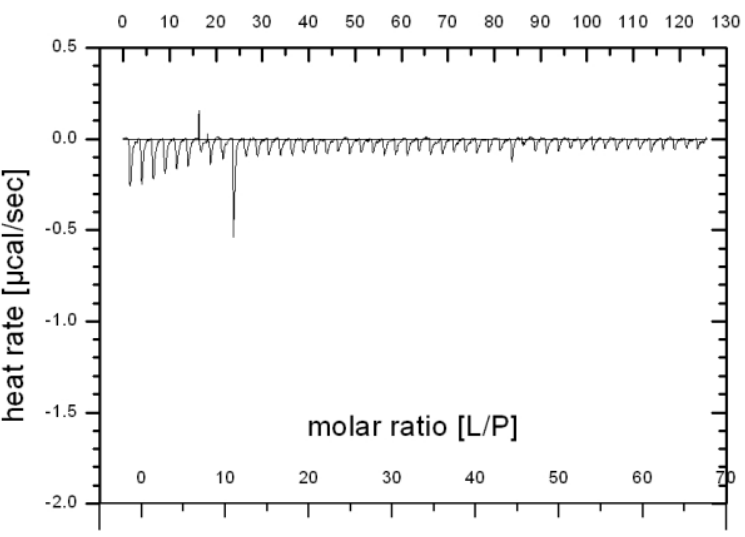



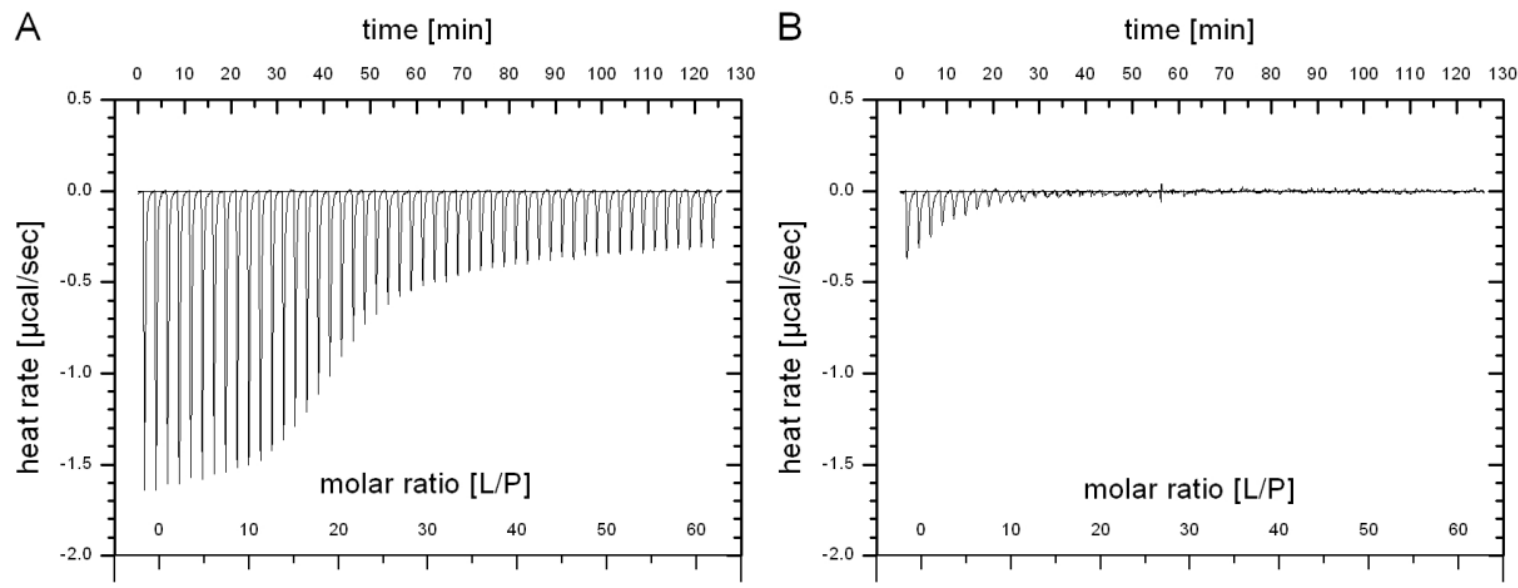

Fig. 6 


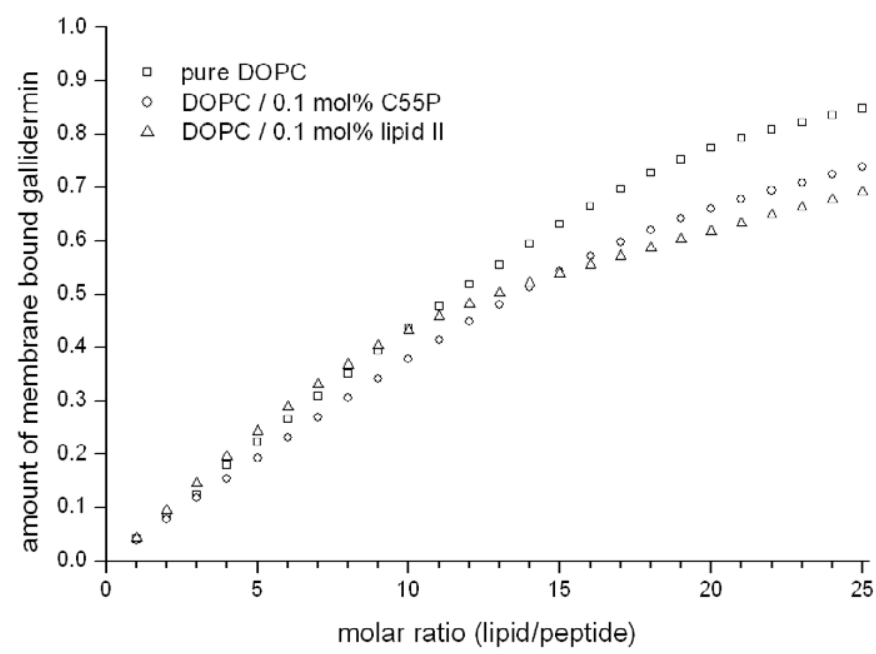

Fig. 7 


\section{Research Highlights}

Al-Kaddah et al.; Analysis of membrane interactions of antibiotic peptides using ITC and biosensor measurements

- antibiotic activities of gallidermin (Gal) and Vancomycin (Van) were simulated

- model membrane approaches are powerful tools to elucidate their mode of action

- Gal and Van have identical affinity to lipid II, but differ in membrane interaction

- Gal targets lipid II in an 1:1 ratio, in synergy inserts and rigidifies the membrane

- SAW technology is excellent to follow peptide membrane interaction in pM range 